\title{
Anterior chest wall resection and sternal body wedge for primary chest wall tumour: reconstruction technique with biological meshes and titanium plates
}

\author{
Alberto Sandri ${ }^{1}$, Giovanni Donati ${ }^{2}$, Carlo Droz Blanc ${ }^{2}$, Victor Auguste Nigra ${ }^{3}$, Matteo Gagliasso ${ }^{1}$, \\ Roberto Barmasse ${ }^{2}$ \\ ${ }^{1}$ Unit of Thoracic Surgery, San Luigi Gonzaga Hospital, University of Turin, Orbassano, Turin, Italy; ${ }^{2}$ Unit of Thoracic Surgery, Parini Hospital, \\ Aosta, Italy; ${ }^{3}$ Department of Thoracic Surgery, Azienda Ospedaliera Universitaria Città della Salute e della Scienza di Torino, University of Turin, \\ Torino, Italy \\ Correspondence to: Alberto Sandri, MD. Unit of Thoracic Surgery, San Luigi Gonzaga Hospital, University of Turin, Orbassano, Turin, Italy. \\ Email: alberto.sandri@icloud.com.
}

Submitted May 28, 2019. Accepted for publication Jun 14, 2019.

doi: $10.21037 /$ jtd.2019.06.45

View this article at: http://dx.doi.org/10.21037/jtd.2019.06.45

\section{Introduction}

Chest wall neoplasms are either primary or metastatic with a malignancy rate of about $50 \%$ percent (1). Chest wall involvement from primary lung neoplasms are uncommon, occurring in approximately $5 \%$ of all primary lung tumours (2). Primary chest wall tumours origin from soft tissues, bone and cartilages; their incidence is $2-5 \%$ in the general population (3). Overall five-year survival after resection of primary chest wall neoplasms is approximately sixty percent; relapse occurs in up to $50 \%$ of patients, with a five-year survival of $17 \%$ (4).

Diagnosis of a chest wall tumour should include a careful patient's history and examination, a computed tomography (CT) scan, nuclear magnetic resonance (RMN) and a positron emission tomography scan (PET). A preoperative histological diagnosis should be obtained by means of fine needle aspiration (FNAB) or excisional biopsy.

Peri- and postoperative complications (mainly pulmonary and infectious) after chest wall resections occur in nearly $25 \%$ of patients, reason why a thorough preoperative assessment of the patient, including pulmonary function tests with DLCo and cardiac evaluation, is highly recommended (5).

In this context, we herewith report our surgical reconstruction technique following an anterior chest wall resection and sternal body wedge for a primary chest wall tumour (chondrosarcoma).

\section{Surgical technique}

Chest wall reconstructions can be particularly challenging depending onto the resection size, site and patient habitus.

The surgical strategy should be carefully analysed preoperatively keeping in mind:

(I) The need of obtaining an oncological radical resection (R0);

(II) A correct identification of the rib(s) and sternal involvement by the tumour by means of a CT scan is the first step (sometimes the tumour is not palpable/visible);

(III) An adequate reconstruction with adequate prosthetic materials, aiming at avoiding lung herniations, to restore the physiological stability, rigidity and synchronisation of the chest wall movements related to breathing and to protect the inner organs.

As an example of this surgical technique, we present the case of a 74 years old gentleman who presented to our Unit for chest pain. On examination, a protruding lesion of the right anterior chest wall was seen and palpated. The patient described an increase of pain and size of the lesion in just few weeks. The CT scan confirmed the presence of an heteroplastic mass $\left(46 \times 42 \times 43 \mathrm{~mm}^{3}\right)$ involving the 4th and 5th ribs anteriorly (Figure 1). An ultrasound guided FNAB was performed and a final diagnosis of mixed 

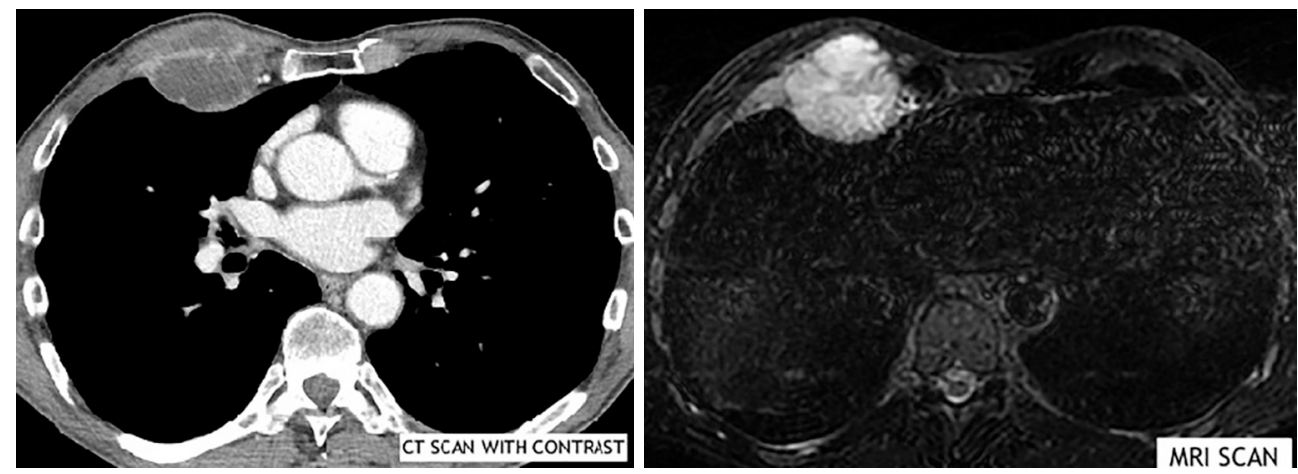

Figure 1 CT scan and MRI showing a chondrosarcoma of the right anterior chest wall, close to the sternal body.

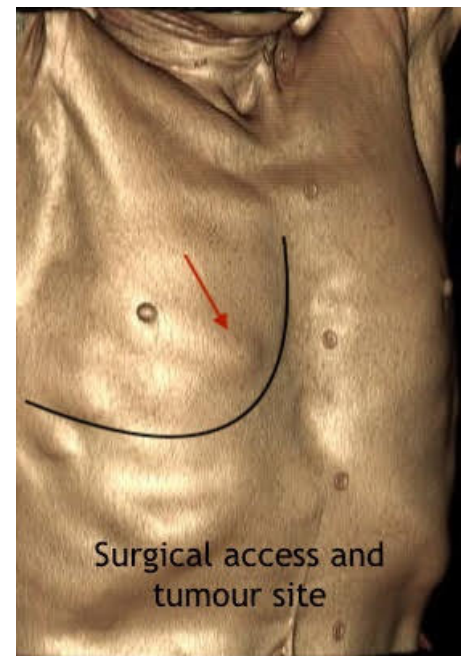

Figure 2 Surgical access-CT scan reconstruction. The arrow indicates the lesion.

chondrosarcoma was obtained. The mass was PET positive with a SUV max of 7. The RMN showed a dimensional increment $\left(54 \times 51 \times 53 \mathrm{~mm}^{3}\right)$ of the tumour, the mass being very close to the sternal body (Figure 1). Surgical indication was given. Usually, the preferred surgical access for these lesions is an anterior thoracotomy, with the skin incision prolonged cutaneously onto the midsternal line (Figure 2). A chest wall resection (ribs III, IV and V) was carried out en-bloc with a sternal body wedge, in order to keep safe margins from the lesion (Figure 3). Reconstruction was performed with a "sandwich technique", by anchoring three titanium plates to the resected ribs and sternal body, in between two layers of biological meshes, one below the plates-to reconstruct the endothoracic cavity and to prevent herniation of the lung and to protect the internal organs-and one above, to reconstruct the muscular plane (Figure 4). A diamond shaped exeresis of the skin and subcutaneous tissues in the site of biopsy (FNAB) was performed (positive for tumour infiltration) (Figure 4). Chest drain was removed in postoperative day 2 (Figure 5).

\section{Discussion}

The characteristics of a successful chest wall reconstruction should aim at restoring the chest wall rigidity, preserving pulmonary mechanics and to protect the intrathoracic organs, avoiding paradox movements of the chest cavity and, possibly, to reduce the thoracic deformity (6).

Several reconstruction techniques and materials have been described in literature over the years. However, the readers would agree that there is not one single material or technique which works best for all reconstructions, but each reconstruction should be approached to with the correct material and technique depending on the need, keeping in mind the requirements elicited by le Roux and Sherma since 1983 (6). The choice of materials available is copious and include synthetic and biological meshes, methyl methacrylate, PTFE (GORE-TEX), titanium plates, allograft and homograft (human and porcine bioprosthetic materials), muscle or omentum flaps and lately $3 \mathrm{D}$ printed customised prosthesis $(7,8)$.

Our preference for reconstruction of anterior chest wall resections with sternal involvement is based onto the use of two different prosthetic materials, a biological mesh (XCM Biologic Tissue Matrix ${ }^{\mathrm{TM}}$ ) and titanium plates $\left(\right.$ MatrixRIB $\left.{ }^{\mathrm{TM}}\right)$. Approaching the reconstruction with two different prosthetic materials should be well pondered since, in case of infection, the need to remove both the prosthetic material is very high. In fact, some authors report 

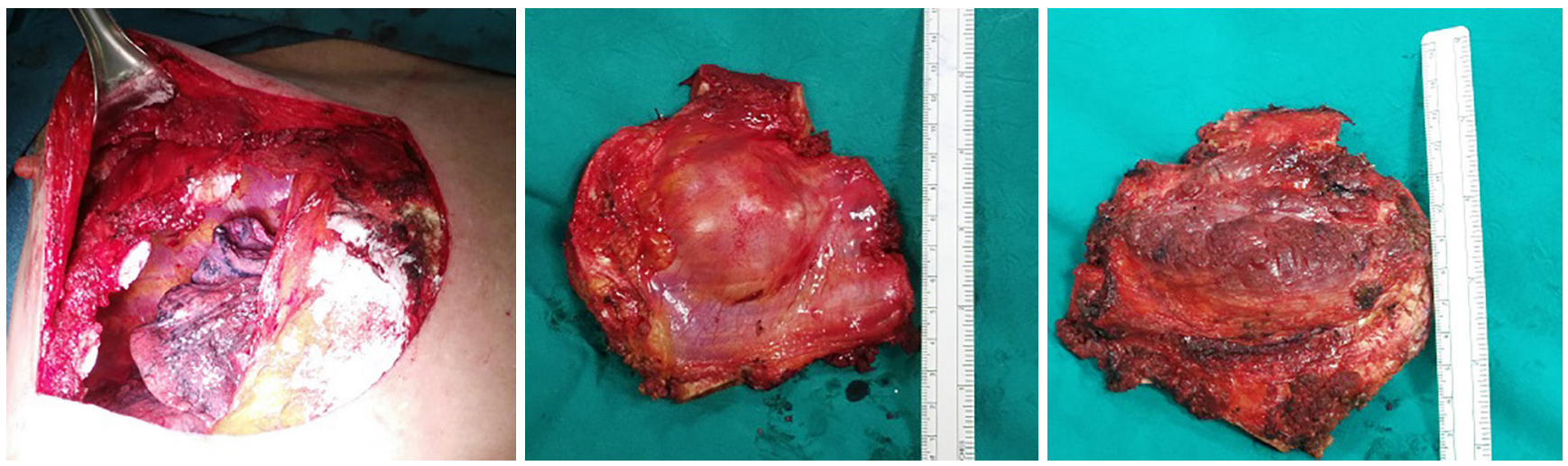

Figure 3 Chest wall resection of chondrosarcoma.
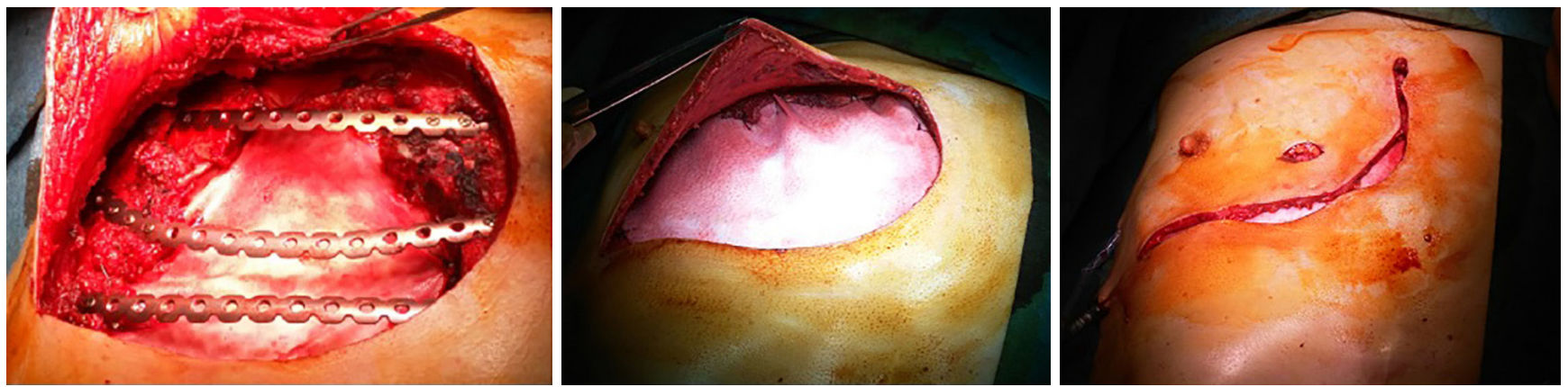

Figure 4 Chest wall reconstruction. "Sandwich technique"- apposition of titanium plates fixed to the ribs and the sternal body, in between two biological meshes.

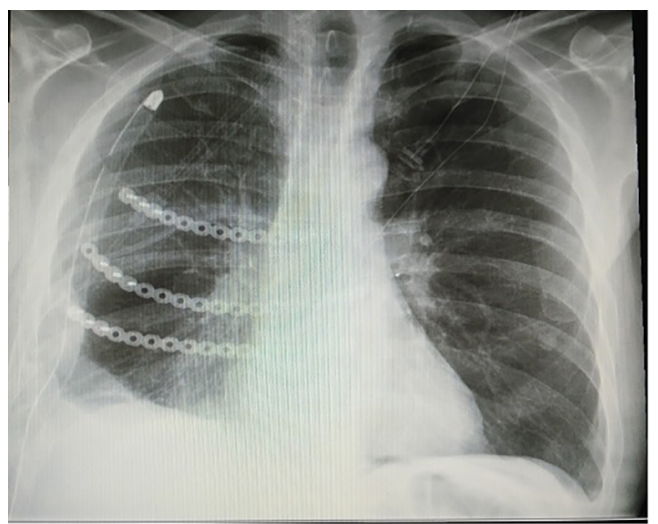

Figure 5 Postoperative CXR. Chest drain was removed in POD 2. CXR, chest X-ray; POD 2, postoperative day 2.

an infection rate of the synthetic meshes comprised between $10 \%$ and $25 \%$ with the need to remove them to resolve the infection (9). The XCM Biologic Tissue Matrix ${ }^{\mathrm{TM}}$ is a biological sterile non-cross-linked three-dimensional matrix, derived from porcine dermis. This mesh is treated with a process that disinfects the tissue, inactivates viruses still granting a cellular re-epithelialization, maintaining the bulk of the natural extra cellular matrix components and minimising damage to tissue architecture. The characteristics of this mesh consent its safe use with a second prosthetic material (titanium plates), it can be easily shaped precisely and grants a good reinforcement over the defect due to its uniform tension strength; also, it creates an ideal substrate which avoids lung herniation and damage, and protects the other inner organs.

The physiological respiratory mechanics which is established through the lung-chest system (compliance/ elastance), is granted if both the lung and the chest wall are intact. The thoracic cage, constituted by the ribs which are anchored anteriorly with their costal cartilages to the sternum and posteriorly to the thoracic vertebrae, are a rigid but plastic system, fundamental in contributing to 


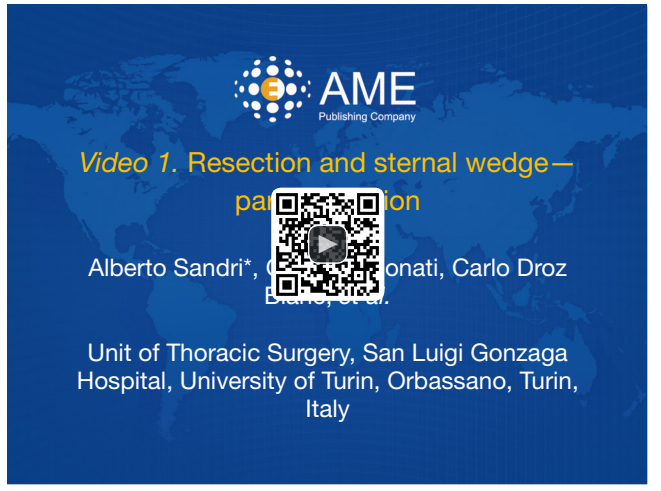

Figure 6 Resection and sternal wedge-part I resection (11). Usually, the preferred surgical access for these lesions is an anterior thoracotomy, with the skin incision prolonged cutaneously onto the midsternal line (Figure 1). In order to obtain a radical resection (R0), the ribs above and below of the involved ones should be resected en-bloc. The pectoral muscles, if not involved by the tumour, are dissected and elevated to access the rib cage. Ribs are then prepared for the resection by scarifying the periosteum of the uninvolved ribs first, with periosteal elevators. Resection is performed with a costotome (Liston) beginning laterally, keeping a safe margin of at least $2 \mathrm{~cm}$ from the tumour. The en-bloc mass with the resected ribs are then elevated medially to access the sternum and to verify possible lung infiltration. Attention should be given to the mammary vessels which should be ligated prior resection. A sternal body wedge is then performed by means of both sternal and oscillating saws, the latter allows a more precise sternotomy. A safe margin of the sternal resection is mandatory. A frozen section of the soft tissues can be of help to confirm margin's clearance. Accurate haemostasis and chest drain placement should be carried out before beginning the reconstruction.

Available online: http://www.asvide.com/watch/33047

stability and breathing synchronicity.

Large defects impairing the thoracic cage integrity, alter the respiratory mechanics severely (paradox breathing), causing respiratory distress and patient instability.

For this reason, it is important to restore its integrity, recreating the original rigidity and stability by means of plates fixed over the margins of the resected ribs and the sternal body itself.

We favour the MatrixRIB ${ }^{\mathrm{TM}}$ Fixation System for our reconstructions; they are precontoured titanium plates which, after a minimal re-modelling following the ribs profile, are fixed to the resected ribs' margins with screws, recreating the

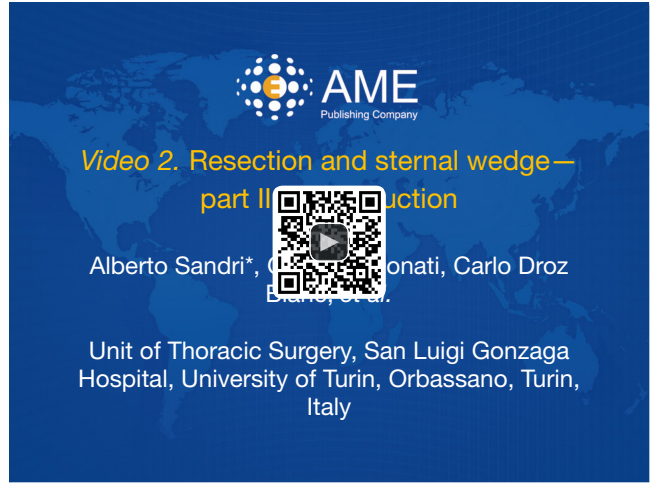

Figure 7 Resection and sternal wedge-part II reconstruction (12). A biologic mesh (XCM Biologic Tissue Matrix ${ }^{\mathrm{TM}}$ ) is sutured to the parietal pleura and to the endothoracic fascia (inferior margin of the resected ribs), ideally reconstructing the endothoracic plane, in order to close the pleural cavity, to prevent lung herniation and possible lung damage through its rubbing/scraping onto the plates. Chest wall reconstruction is then carried out with modelling the precontoured titanium plates to fit the original rib profile (MatrixRIB ${ }^{\mathrm{TM}}$ Fixation System), and by locking them with screws onto the ribs and the sternal body, thus granting rigidity, stability of the chest wall and avoiding paradox movements. Especially in large anterior chest wall resections and/or in lean patients, another biological mesh to reconstruct the muscle plain and the fascia over the plates is advisable, as presented in the video. A diamond shaped exeresis of the skin and subcutaneous tissues in the site of FNAB, is highly recommended.

Available online: http://www.asvide.com/watch/33048

anatomical and physiological appearance of the thoracic cage.

The locking screws instead of rib clips fixation bar are, in our opinion, more advisable, since: (I) are likely to be more tolerated postoperatively because of less pain due to minor costal bundle stress and (II) are more prone to a less postoperative dislocation/breakage rate $(7,10)$.

As seen in the videos of our case (Figures 6,7), because of the patient's poor tissue habitus, we implanted and fixed to the extrathoracic soft tissues and the pectoralis major muscle (previously disconnected from the rib cage), over the titanium bars, a second shaped XCM Biologic Tissue Matrix ${ }^{\mathrm{TM}}$ mesh ("sandwich technique").

Regardless of the technique used to establish skeletal stability, full tissue coverage of the prosthesis is mandatory, using direct suture, skin grafts, local advancement flaps, pedicled myocutaneous flaps or free flaps (13). 


\section{Acknowledgments}

None.

\section{Footnote}

Conflicts of Interest: The authors have no conflicts of interest to declare.

Ethical Statement: The authors are accountable for all aspects of the work in ensuring that questions related to the accuracy or integrity of any part of the work are appropriately investigated and resolved. Written informed consent was obtained from the patient for publication of this manuscript and any accompanying images.

\section{References}

1. D'Addario G, Früh M, Reck M, et al. Metastatic non-small-cell lung cancer: ESMO Clinical Practice Guidelines for diagnosis, treatment and follow-up. Ann Oncol 2010;21 Suppl 5:v116-9.

2. Filosso PL, Sandri A, Guerrera F, et al. Primary lung tumors invading the chest wall. J Thorac Dis 2016;8:S855-62.

3. Shah AA, D'Amico TA. Primary chest wall tumors. J Am Coll Surg 2010;210:360-6.

4. King RM, Pairolero PC, Trastek VF, et al. Primary chest wall tumors: factors affecting survival. Ann Thorac Surg

Cite this article as: Sandri A, Donati G, Blanc CD, Nigra VA, Gagliasso M, Barmasse R. Anterior chest wall resection and sternal body wedge for primary chest wall tumour: reconstruction technique with biological meshes and titanium plates. J Thorac Dis 2020;12(1):17-21. doi: 10.21037/jtd.2019.06.45
1986;41:597-601.

5. Spicer JD, Shewale JB, Antonoff MB, et al. The Influence of Reconstructive Technique on Perioperative Pulmonary and Infectious Outcomes Following Chest Wall Resection. Ann Thorac Surg 2016;102:1653-9.

6. le Roux BT, Shama DM. Resection of tumors of the chest wall. Curr Probl Surg 1983;20:345-86.

7. Sanna S, Brandolini J, Pardolesi A, et al. Materials and techniques in chest wall reconstruction: a review. J Vis Surg 2017;3:95.

8. $\mathrm{Wu} \mathrm{Y}$, Chen $\mathrm{N}, \mathrm{Xu} \mathrm{Z}$, et al. Application of $3 \mathrm{D}$ printing technology to thoracic wall tumor resection and thoracic wall reconstruction. J Thorac Dis 2018;10:6880-90.

9. Daigeler A, Druecke D, Hakimi M, et al. Reconstruction of the thoracic wall-long-term follow-up including pulmonary function tests. Langenbecks Arch Surg 2009;394:705-15.

10. Muthialu N, McIntyre D, McIntosh N, et al. Disturbingly high fracture rate of STRATOS bars in pectus corrections. Eur J Cardiothorac Surg 2019;55:300-3.

11. Sandri A, Donati G, Blanc CD, et al. Resection and sternal wedge-part I resection. Asvide 2020;7:003. Available online: http://www.asvide.com/watch/33047

12. Sandri A, Donati G, Blanc CD, et al. Resection and sternal wedge-part II reconstruction. Asvide 2020;7:004. Available online: http://www.asvide.com/watch/33048

13. Seder CW, Rocco G. Chest wall reconstruction after extended resection. J Thorac Dis 2016;8:S863-71. 